\title{
Sniff nasal inspiratory pressure in patients with chronic obstructive pulmonary disease
}

\author{
C. Uldry, J.P. Janssens, B. de Muralt, J.W. Fitting
}

Sniff nasal inspiratory pressure in patients with chronic obstructive pulmonary disease. C. Uldry, J.P. Janssens, B. de Muralt, J.W. Fitting. CERS Journals Ltd 1997.

ABSTRACT: In subjects with normal lung mechanics, inspiratory muscle strength can be reliably and easily assessed by the sniff nasal inspiratory pressure (SNIP), which is the pressure measured in an occluded nostril during a maximal sniff performed through the contralateral nostril. The aim of this study was to assess the validity of the SNIP in patients with chronic obstructive pulmonary disease (COPD), where pressure transmission from alveoli to upper airways is likely to be dampened.

Twenty eight patients with COPD were studied (mean forced expiratory volume in one second $($ FEV1) $=36 \%$ of predicted). The SNIP and the sniff oesophageal pressure (sniff $P_{0 e s}$ ) were measured simultaneously during maximal sniffs, and were compared to the maximal inspiratory pressure obtained against an occlusion (MIP). All measurements were performed from functional residual capacity in the sitting position.

The ratio SNIP/sniff $P_{0 e s}$ was 0.80 , and did not correlate with the degree of airflow limitation. The ratio MIP/sniff $P_{0}$ es was 0.87 , and the ratio SNIP/MIP was 0.97. Inspiratory muscle weakness, as defined by a low sniff $P_{0 e s,}$ was present in 17 of the 28 patients. A false diagnosis of weakness was made in eight patients when MIP was considered alone, in four when SNIP was considered alone, and in only three patients when MIP and SNIP were combined.

We conclude that both the sniff nasal inspiratory pressure and the maximal inspiratory pressure moderately underestimate sniff oesophageal pressure in chronic obstructive pulmonary disease. Although suboptimal in this condition, the sniff nasal inspiratory pressure appears useful to complement the maximal inspiratory pressure for assessing inspiratory muscle strength in patients with chronic obstructive pulmonary disease.

Eur Respir J 1997; 10: 1292-1296.
Division de Pneumologie, Centre Hospitalier Universitaire Vaudois, Lausanne, Switzerland, and Centre de Réhabilitation Respiratoire, Hôpital de Rolle, Switzerland.

Address: J.W. Fitting

Division de Pneumologie CHUV

1011 Lausanne

Switzerland

Keywords: Obstructive lung diseases respiratory muscles respiratory pressures

Received: January 261996 Accepted after revision February 21997
Classically, the strength of the inspiratory muscles is assessed by the maximal inspiratory pressure (MIP or $P \mathrm{I}, \max )$, i.e. the pressure measured at the mouth and sustained for $1 \mathrm{~s}$ during a maximal inspiratory effort performed against an occlusion [1]. However, this type of maximal effort is closely dependent on the subject's collaboration. Because the manoeuvre is demanding and unpleasant, the results are prone to considerable variation. Thus, low values may reflect true muscle weakness, but may also result from a lack of motivation and co-ordination. Using short, maximal sniffs has been shown to be a valuable alternative method [2]. Inspiratory muscle strength is often better reflected by oesophageal pressure during a maximal sniff (sniff $P$ oes) than by MIP [3]. However, sniff $P_{\text {oes }}$ is an invasive method requiring an oesophageal balloon catheter system, which limits its clinical usefulness.

The sniff nasal inspiratory pressure (SNIP) provides a noninvasive measurement of inspiratory muscle strength. The SNIP is measured through a plug occluding one nostril during a sniff performed through the contralateral nostril. In normal subjects and in patients with neuromuscular or skeletal disorders, the mean ratio SNIP/ sniff $P$ oes was 0.92 [4]. A recent study showed that SNIP was higher than MIP in healthy subjects, and that normal values for SNIP could be predicted from age and sex [5]. Abnormal lung and airway mechanics are likely to impair pressure transmission along the airways during brief dynamic manoeuvres.

Therefore, the aim of the present study was to assess the validity of SNIP for estimating inspiratory muscle strength in patients with chronic obstructive pulmonary disease (COPD).

\section{Methods}

\section{Subjects}

Twenty eight patients with COPD were studied in their stable clinical state. None was accustomed to performing the manoeuvres and none had rhinitis or previous nasal surgery. Their mean forced expiratory volume 
in one second (FEV1) was $36 \%$ of predicted, and their mean $\mathrm{FEV} 1 /$ forced vital capacity (FVC) ratio was $53 \%$ of predicted. The physical characteristics of the subjects are presented in table 1 . The study was approved by the Ethics Committee of our hospital and all subjects gave their informed consent.

\section{Measurements}

Total lung capacity (TLC) and residual volume (RV) were measured by plethysmography, and forced expiratory flow rates were measured by mass flow sensor (SensorMedics 6200 Autobox; Yorba Linda, CA, USA). The SNIP was measured through a plug occluding one nostril during a sniff performed through the contralateral nostril, as described previously [4]. The plug was made of waxed ear plugs (Calmor, Neuhausen am Rheinfall, Switzerland) hand-fastened around the tip of a catheter. Oesophageal pressure (sniff $P_{\text {oes}}$ ) was measured with a $5 \mathrm{~cm}$ long balloon, filled with $0.5 \mathrm{~mL}$ of air and positioned in the mid-oesophagus. The nasal plug and oesophageal balloon were connected to two pressure transducers (MicroSwitch 126 PC, linearity 0-350 $\mathrm{cmH}_{2} \mathrm{O}$; Honeywell, Freeport, IL, USA) via identical $100 \mathrm{~cm}$ polyethylene catheters, and the pressure was recorded on paper (Gould EasyGraph, Valley View, OH, USA).

The subjects were studied in the sitting position without prior training. They were asked to breathe normally through the patent nostril with closed mouth, and to perform 10 maximal short and sharp sniffs from functional residual capacity (FRC), each separated by $30 \mathrm{~s}$. FRC was identified as the end of expiration during quiet breathing. No visual feedback was provided. All manoeuvres were recorded. If the 10th sniff gave the highest value, additional attempts were recorded until no further increase in pressure was seen. The transducer was calibrated before each measurement session using a water manometer.

MIP sustained over $1 \mathrm{~s}$ was measured with a standard flanged mouthpiece connected to a portable mouth pressure Meter (Chest Scientific Instrument Ltd, Westerham, UK). The subjects were studied in the sitting position, breathing through the nose with the mouth occluded by the mouthpiece. They were asked to occlude their nose and to perform four maximal inspiratory efforts from FRC, each separated by $1 \mathrm{~min}$. If the greatest pressure was obtained with the fourth manoeuvre, additional measurements were made until no further increase was seen.

Table 1. - Physical characteristics of 28 patients with COPD

\begin{tabular}{lcc}
\hline Gender M/F & $22 / 6$ & \\
Age yrs & $63 \pm 12$ & $(22-80)$ \\
Height m & $1.7 \pm 0.1$ & $(1.5-1.8)$ \\
Weight kg & $69 \pm 14$ & $(43-99)$ \\
FEV1 \% pred & $36 \pm 13$ & $(16-69)$ \\
FEV1/FVC \% pred & $53 \pm 12$ & $(32-73)$ \\
TLC \% pred & $112 \pm 18$ & $(80-143)$ \\
RV/TLC \% pred & $167 \pm 32$ & $(124-218)$ \\
\hline
\end{tabular}

Values are presented as mean $\pm \mathrm{SD}$, and range in parenthesis, COPD: chronic obstructive pulmonary disease; M: male; F: female; FEV1: forced expiratory volume in one second; FVC: forced vital capacity; TLC: total lung capacity; RV: residual volume; $\%$ pred: percentage of predicted value.

\section{Data analysis}

SNIP, sniff $P_{\text {oes }}$ and MIP represent the amplitudes of pressure changes, and are expressed in absolute values, as mean (SD). For each patient, the highest values of SNIP, sniff $P_{\text {oes }}$ and MIP were used for analysis. The correlation coefficient (r) between SNIP and sniff $P_{\text {oes }}$ was calculated, and linear regression analysis of the two measurements was performed. The agreement between SNIP and sniff $P_{\text {oes, }}$ MIP and sniff $P_{\text {oes, }}$ and SNIP and MIP was assessed by the method of differences against the means, according to Bland and Altman [6]. The relationships between the ratio SNIP/sniff $P_{\text {oes }}$ and FEV1 and $\mathrm{FEV} 1 / \mathrm{FVC}$ were assessed by linear regression analysis. A p-value of less than 0.05 was considered significant.

\section{Results}

The maximal values of SNIP, sniff $P_{\text {oes, }}$ and MIP are presented in table 2 . Regression analysis showed that SNIP $\left(\mathrm{cmH}_{2} \mathrm{O}\right)=0.88$ sniff $P$ oes $-5.1(\mathrm{r}=0.92 ; \mathrm{p}<0.0001)$. Figure 1a presents the relationship between SNIP and sniff $P_{\text {oes. }}$ The mean (SD) SNIP/sniff $P$ oes ratio was 0.80 (0.13). Figure $1 \mathrm{~b}$ presents a plot of the difference between SNIP and sniff $P_{\text {oes }}$ against their mean. The mean difference (d) was -14 (10) $\mathrm{cmH}_{2} \mathrm{O}$. The limits of agreement were:

$$
\begin{aligned}
& \text { upper limit: } d+2 \mathrm{SD},-14+20=6 \mathrm{cmH}_{2} \mathrm{O} \\
& \text { lower limit: } d-2 \mathrm{sD},-14-20=-34 \mathrm{cmH}_{2} \mathrm{O}
\end{aligned}
$$

No correlation was found between the SNIP/sniff $P_{\text {oes }}$ ratio and the FEV1 (\% pred) $(\mathrm{p}=0.7)$ or the $\mathrm{FEV} 1 / \mathrm{FVC}$ ratio $(\%$ pred $)(\mathrm{p}=0.8)$.

Figure 2a presents the relationship between MIP and sniff $P_{\text {oes. }}$ The mean (SD) MIP/sniff $P$ oes ratio was 0.87 (0.23). Figure $2 \mathrm{~b}$ presents a plot of the difference between MIP and sniff $P$ oes against their mean. The mean difference (d) was -11 (21) $\mathrm{cmH}_{2} \mathrm{O}$. The limits of agreement were:

$$
\begin{aligned}
& \text { upper limit: } d+2 \mathrm{sD},-11+42=31 \mathrm{cmH}_{2} \mathrm{O} \\
& \text { lower limit: } d-2 \mathrm{sD},-11-42=-53 \mathrm{cmH}_{2} \mathrm{O}
\end{aligned}
$$

Figure 3a presents the relationship between SNIP and MIP. The mean (SD) SNIP/MIP ratio was 0.97 (0.28). Figure $3 \mathrm{~b}$ presents a plot of the difference between SNIP and MIP against their mean. The mean difference (d) was $-3(18) \mathrm{cmH}_{2} \mathrm{O}$. The limits of agreement were:

$$
\begin{aligned}
& \text { upper limit: } d+2 \mathrm{SD},-3+36=33 \mathrm{cmH}_{2} \mathrm{O} \\
& \text { lower limit: } d-2 \mathrm{sD},-3-36=-39 \mathrm{cmH}_{2} \mathrm{O}
\end{aligned}
$$

\begin{tabular}{|c|c|}
\hline SNIP $\mathrm{cmH}_{2} \mathrm{O}$ & $58 \quad(24)$ \\
\hline sniff $P_{\text {oes }} \quad \mathrm{cmH}_{2} \mathrm{O}$ & $72(25)$ \\
\hline MIP $\mathrm{cmH}_{2} \mathrm{O}$ & 61 (18) \\
\hline SNIP/sniff $P$ oes & $0.80 \quad(0.13)$ \\
\hline MIP/sniff $P_{\text {oes }}$ & $0.87 \quad(0.23)$ \\
\hline SNIP/MIP & $0.97 \quad(0.28)$ \\
\hline
\end{tabular}

Table 2. - Values of sniff nasal inspiratory pressure (SNIP), sniff oesophageal pressure (sniff $P_{\text {oes}}$ ), and maximal inspiratory pressure (MIP) in 28 patients with COPD

Values are presented as mean, and SD in parenthesis. COPD: chronic obstructive pulmonary disease. 

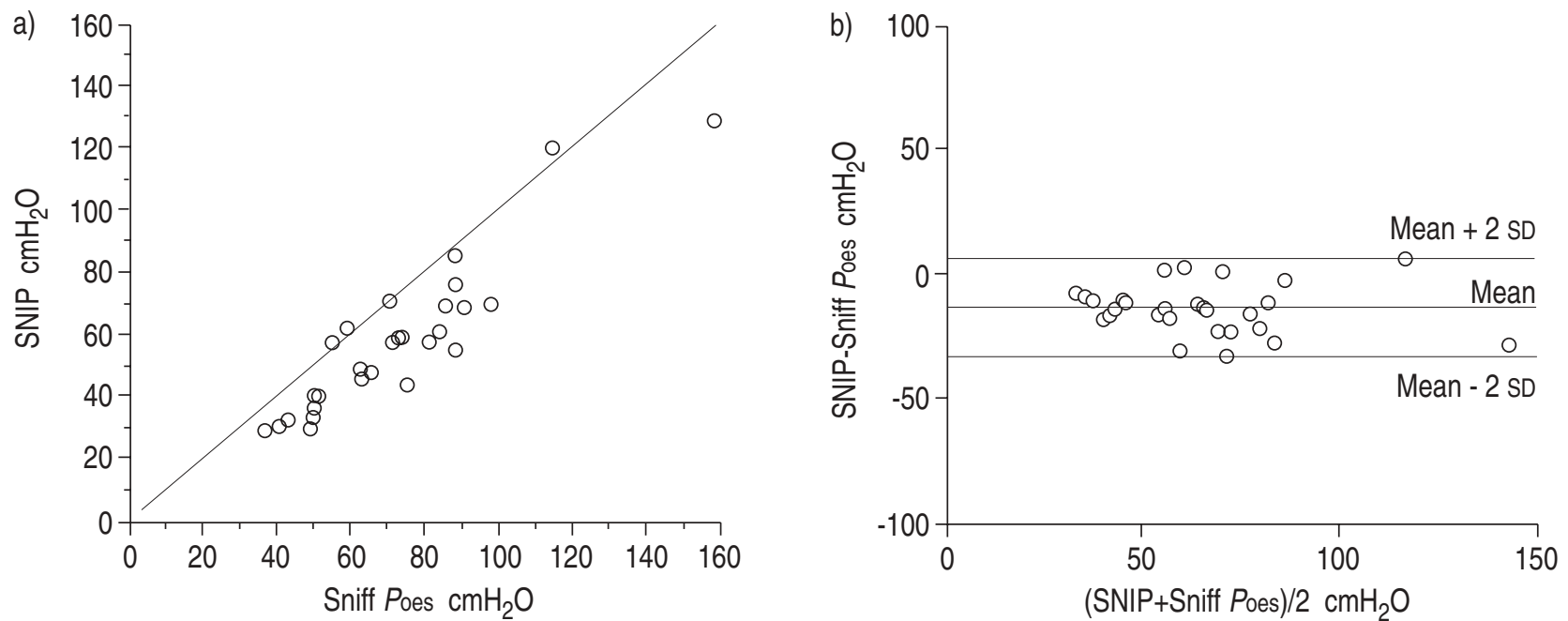

Fig. 1. - a) Relationship between sniff nasal inspiratory pressure (SNIP) and sniff oesophageal pressure (sniff $P_{\text {oes}}$ ) in 28 patients with chronic obstructive pulmonary disease (COPD). The line of identity is shown. b) Difference between SNIP and sniff $P$ oes against the mean of these two variables in 28 patients with COPD.
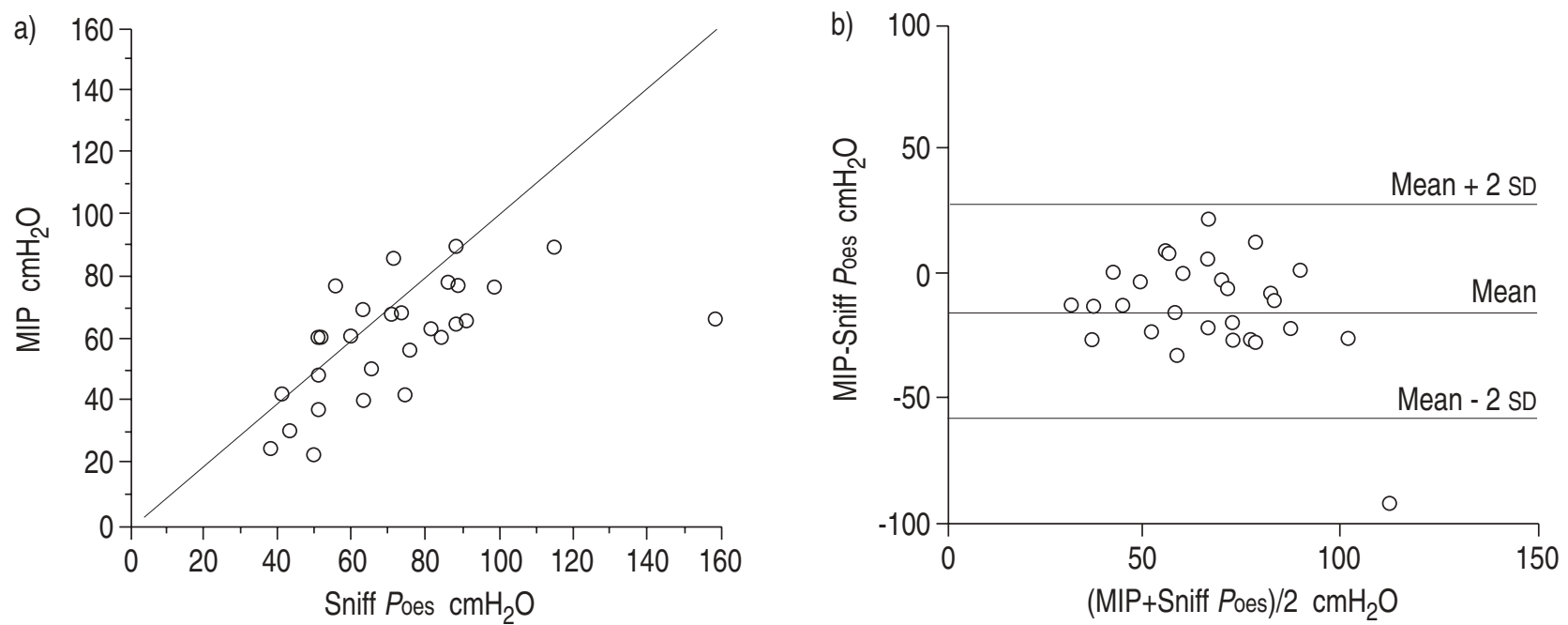

Fig. 2. - a) Relationship between maximal inspiratory pressure (MIP) and sniff oesophageal pressure (sniff $P$ oes) in 28 patients with chronic obstructive pulmonary disease (COPD). The line of identity is shown. b) Difference between MIP and sniff Poes against the mean of these two variables in 28 patients with COPD.
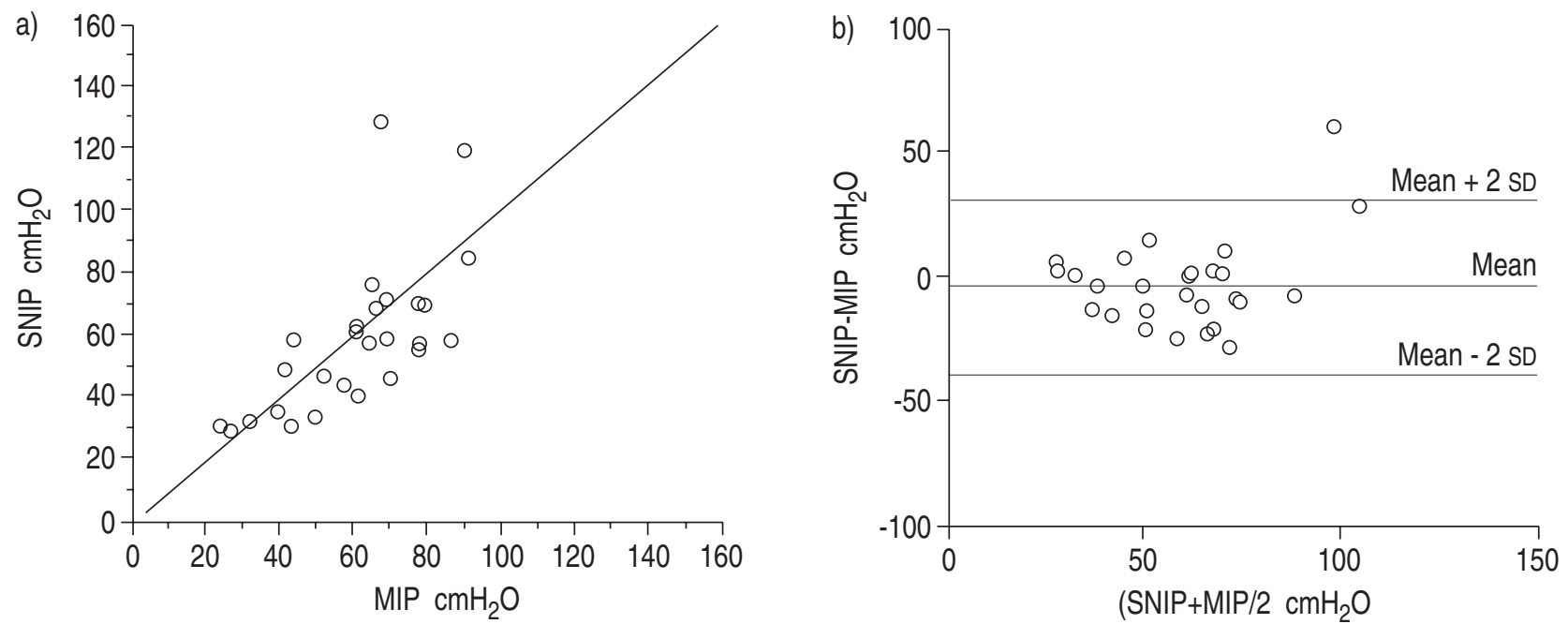

Fig. 3. - a) Relationship between sniff nasal inspiratory pressure (SNIP) and maximal inspiratory pressure (MIP) in 28 patients with chronic obstructive pulmonary disease (COPD). The line of identity is shown. b) Difference between SNIP and MIP against the mean of these two variables in 28 patients with COPD. 
Using a low sniff $P_{\text {oes }}\left(<80 \mathrm{cmH}_{2} \mathrm{O}\right.$ for males, $<70$ $\mathrm{cmH}_{2} \mathrm{O}$ for females) as the criterion [7], inspiratory muscle weakness was present in 17 of the 28 patients. A false diagnosis of weakness was made in eight patients when MIP was low and considered alone $\left(<80 \mathrm{cmH}_{2} \mathrm{O}\right.$ for males, $<70 \mathrm{cmH}_{2} \mathrm{O}$ for females [7]), and in four patients when SNIP was low and considered alone $(<70$ $\mathrm{cmH}_{2} \mathrm{O}$ for males, $<60 \mathrm{cmH}_{2} \mathrm{O}$ for females [5, 7]). Only 3 of the 28 patients were falsely diagnosed as weak when SNIP and MIP were combined.

\section{Discussion}

The maximal sniff manoeuvre has been shown to be both easy to perform and a reliable test of inspiratory muscle strength. Thus, LAROCHE et al. [2] reported that inspiratory muscle strength was most often better reflected by sniff $P_{\text {oes }}$ than by MIP. The SNIP, i.e. the nasal pressure measured through a plug occluding one nostril during a maximal sniff performed through the contralateral nostril, provides a reliable and noninvasive estimate of $P_{\text {oes }}$ both in healthy subjects and patients with neuromuscular or skeletal disorders [4]. During the sniff, the nasal valve located in the first $2.5 \mathrm{~cm}$ from the external orifice collapses when a critical transnasal pressure of $10-15 \mathrm{cmH}_{2} \mathrm{O}$ is reached $[8,9]$. In subjects with normal lung and airway mechanics, there is only a small pressure gradient between the alveoli and extra-thoracic airways located proximally to the point of collapse. Normal values have been established for the SNIP, which can be predicted from age by a first degree equation for each sex [5]. Thus, the SNIP represents a simple and useful test that can be included in an incremental approach to assess respiratory muscle strength [7].

So far, the validity and usefulness of the SNIP has only been assessed in normal subjects and in patients with neuromuscular or skeletal disorders [4]. In the present study, we found that the SNIP underestimated sniff $P$ oes in patients with COPD, with a mean SNIP/sniff $P$ oes ratio of 0.80 . The difference between SNIP and sniff $P$ oes was consistent among patients, as illustrated by the relatively close limits of agreement in figure $1 \mathrm{~b}$. This difference could be expected in view of the short and dynamic character of the sniff. Similar observations have been made with the occlusion pressure, i.e. the pressure change measured at the mouth $0.1 \mathrm{~s}$ after the onset of inspiration (P0.1). MARAZZINI et al. [10] measured mouth and oesophageal occlusion pressure during $\mathrm{CO}_{2}$ rebreathing in normal subjects and in patients with COPD. The normal subjects showed similar responses in terms of mouth and oesophageal occlusion pressure. In contrast, the patients with COPD demonstrated a smaller response of occlusion pressure measured at the mouth than in the oesophagus. Murciano et al. [11] compared oesophageal and tracheal occlusion pressure in acutely ill patients with COPD who were either intubated or tracheotomized. They found no difference between occlusion pressure measured in the trachea and in the oesophagus. In a few of these patients, they also showed that mouth occlusion pressure was approximately half of oesophageal occlusion pressure during mouth breathing, with tracheostomy obstructed. In such a circumstance, the transmission of pressure changes from the alveoli to the mouth depends on a time constant, which is the product of airway resistance and upper airway compliance.

These observations demonstrate that pressure transmission from the alveoli to the mouth is dampened by an increased time constant in patients with COPD. This phenomenon is of prime importance for the measurements of short events, such as occlusion pressure or sniff pressure. Accordingly, the underestimation of sniff $P_{\text {oes }}$ by the SNIP might be expected to vary with the degree of airflow limitation. This did not appear to be the case in this study, as no correlation existed between the ratio SNIP/sniff $P$ oes and either FEV1 or FEV1/FVC. However, it should be noted that these indices describe expiratory flow, whereas the sniff is an inspiratory manoeuvre.

Thus, the SNIP underestimates true inspiratory muscle strength in patients with COPD. The question then arises of the usefulness of this test in conditions where its validity is suboptimal. In fact, MIP, the test classically used in this instance, also underestimated inspiratory muscle strength in these patients: the ratio MIP/ sniff $P$ oes was 0.87 . This observation is in accordance with a previous study showing MIP to be lower than sniff $P$ oes in patients suspected of muscle weakness [2]. In patients with obstructive airway disease, as in other subjects, the reason probably lies in the difficulty of the sustained maximal inspiratory effort compared to the ease of the sniff manoeuvre. The limits of agreement between MIP and either SNIP or sniff $P_{\text {oes }}$ were wide, underlining the distinct character of these two manoeuvres.

Our study shows that SNIP and MIP are suboptimal in COPD because they both underestimate inspiratory muscle strength in these patients. However, even though the mean difference between SNIP and MIP is small, the limits of agreement between them are wide, as illustrated by figure $3 \mathrm{~b}$. This means that the two tests complement one another, the highest pressure being given by MIP in some patients and by SNIP in others. In this study, the number of false diagnoses of weakness was less with the SNIP than with the MIP, and was the lowest when the two tests were combined.

We conclude that the sniff nasal inspiratory pressure moderately underestimates sniff oesophageal pressure and, thus, inspiratory muscle strength, in patients with chronic obstructive pulmonary disease. On average, the underestimation of inspiratory muscle strength is similar with the measurement of sniff nasal inspiratory pressure and maximal inspiratory pressure, but each of these tests may prove better than the other in individual patients. In spite of its suboptimal validity in this condition, the sniff nasal inspiratory pressure may, therefore, usefully complement the maximal inspiratory pressure in assessing inspiratory muscle strength in patients with chronic obstructive pulmonary disease.

\section{References}

1. Black LF, Hyatt RE. Maximal respiratory pressures: normal values and relationship to age and sex. Am Rev Respir Dis 1969; 99: 696-702.

2. Laroche CM, Mier AK, Moxham J, Green M. The value of sniff oesophageal pressures in the assessment of 
global inspiratory muscle strength. Am Rev Respir Dis 1988; 138: 598-603.

3. Koulouris N, Mulvey DA, Laroche CM, Sawicka EH, Green M, Moxham J. The measurement of inspiratory muscle strength by sniff esophageal, nasopharyngeal, and mouth pressure. Am Rev Respir Dis 1989; 139: 641-646.

4. Héritier F, Rahm F, Pasche P, Fitting JW. Sniff nasal inspiratory pressure: a noninvasive assessment of inspiratory muscle strength. Am J Respir Crit Care Med 1994; 150: $1678-1683$.

5. Uldry C, Fitting JW. Maximal values of sniff nasal inspiratory pressure in healthy subjects. Thorax 1995; 50: 371-375.

6. Bland JM, Altman DG. Statistical methods for assessing agreement between two methods of clinical measurement. Lancet 1986; i: 307-310.
7. Polkey MI, Green M, Moxham J. Measurement of respiratory muscle strength. Thorax 1995; 50: 11311135.

8. Bridger GP, Proctor DF. Maximal nasal inspiratory flow and nasal resistance. Ann Otol Rhinol Laryngol 1970; 79: 481-488.

9. Haight JSJ, Cole P. The site and function of the nasal valve. Laryngoscope 1983; 93: 49-55.

10. Marazzini L, Cavestri R, Gori D, Gatti L, Longhini E. Difference between mouth and esophageal pressure during $\mathrm{CO}_{2}$ rebreathing in chronic obstructive pulmonary disease. Am Rev Respir Dis 1978; 118: 1027-1033.

11. Murciano D, Aubier M, Bussi S, Derenne J-P, Pariente R, Milic-Emili J. Comparison of esophageal, tracheal and mouth occlusion pressure in patients with chronic obstructive pulmonary disease. Am Rev Respir Dis 1982; 126: 837-841. 